\title{
Energy Resource Scheduling with Multiple Iterations for the Validation of Demand Response Aggregation
}

\author{
João Spínola, Pedro Faria, Zita Vale \\ Polytechnic of Porto \\ Porto, Portugal \\ \{jafps, pnf, zav\}@isep.ipp.pt
}

GECAD - Research Group on Intelligent Engineering and Computing for Advanced Innovation and Development, ISEP/IPP -

\begin{abstract}
Flexibility aggregators are becoming a trend in European energy markets, joining several consumers and smallsize distributed generators to their portfolio, enabling market participation. Also, the interest growth in clean energy resources and in smart grid concepts such as demand response and communication infrastructures, has led to a facilitation in the integration of these flexibility resources. In this paper, it is proposed a methodology that supports the aggregator in its energy management and resources scheduling. The work focuses on a rescheduling method that uses aggregation and remuneration processes to define new tariffs for consumers participating in a load curtailment demand response program. Aggregation is performed using the clustering algorithm, $k$ means, while the remuneration process defines a tariff per group formed by computing the arithmetic average of the consumer's prices belonging to each group.
\end{abstract}

Index Terms-Aggregator; Demand response; Distributed energy resources; optimization; rescheduling.

\section{INTRODUCTION}

The continuous appearance of energy aggregator models and companies in Europe has led to several challenges in the management of distributed energy resources. Moreover, when considering that these can participate in energy markets, the number of activities developed by an aggregator implicates distinct concepts, such as, demand response and distributed generation [1], [2]. The first is defined as load profile modifications in response to monetary incentives and/or price signals. The latter is related to all small-size generators that are connected to distribution networks. Both concepts represent flexibility resources that can provide useful services to the power system [3]-[6].

The normal procedure of the aggregator is to create a summed virtual amount based on distributed energy resources and, in the flexibility that these provide, that enables its participation in energy markets. From this participation, the aggregator must be able to obtain the necessary revenues that guarantee the payment of the used resources, and profit from this operation. The analysis of market participation by the aggregator, must evaluate the reliability of using the resources

This work has been developed under the EUREKA - ITEA2 Project FUSE-IT (ITEA-13023), Project GREEDI (ANI|P2020 17822), and has received funding from FEDER Funds through COMPETE program and from National Funds through FCT under the project UID/EEA/00760/2013.
[7], [8]. There are several markets that can benefit from the aggregator's participation, mainly, the ancillary services market. This market is dedicated to the gathering of fastresponse flexibility to be used in the correction of system parameters, mainly, frequency and voltage [9], [10].

During the operation of the network, constant variations occur due to the connection and disconnection of various types of loads. These variations cause the network parameters, frequency and voltage, to be unbalanced in some periods which leads to a poor energy quality and delivery to the enduser. In this way, the ancillary services market intends to gather flexibility of operation to quickly correct the network parameters when these are out of an appropriate range [11], [12]. For the aggregator to participate in these markets, a scheduling of resources must be performed to obtain the contributions of each one.

The present paper is built upon previous works, [13], [14], that provided the aggregator with aggregation and remuneration features, but an analysis was not made of the influence that these have in the aggregator's scheduling of resources. Thus, the present paper proposes a methodology to support the activities of an aggregator, considering demand response flexibility aggregation and remuneration based on a group tariff. After an initial scheduling of resources, aggregation and remuneration processes, a new set of load curtailment prices are obtained, followed by a second scheduling based on those new prices. The aggregator is then able to evaluate the two scenarios (first scheduling and second scheduling considering aggregation and remuneration processes) and decide on whether adopt one solution or the other, according to the operation costs obtained.

This introductory section provided the theoretical basis for the proposed methodology, and what it intends to improve regarding the aggregator's operation. The next section, explains the components and processes that compose the proposed methodology, including a detailed explanation of each stage, namely, the mathematical formulation for the scheduling of resources, and the aggregation and remuneration processes. Further on, Section III presents the case study used 
to verify the usefulness of the proposed methodology. Section IV shows the results obtained in the simulation of the case study, and finally, Section VI outlines the conclusions achieved in the present work.

\section{PROPOSED METHODOLOGY}

This section details the proposed methodology regarding the scheduling of resources by an aggregator, based on a rescheduling approach that considers aggregation and remuneration methods. The proposed methodology is shown in Figure 1. The resources available for scheduling by the aggregator are: external suppliers, distributed generation and consumers participating in a curtailment demand response program.

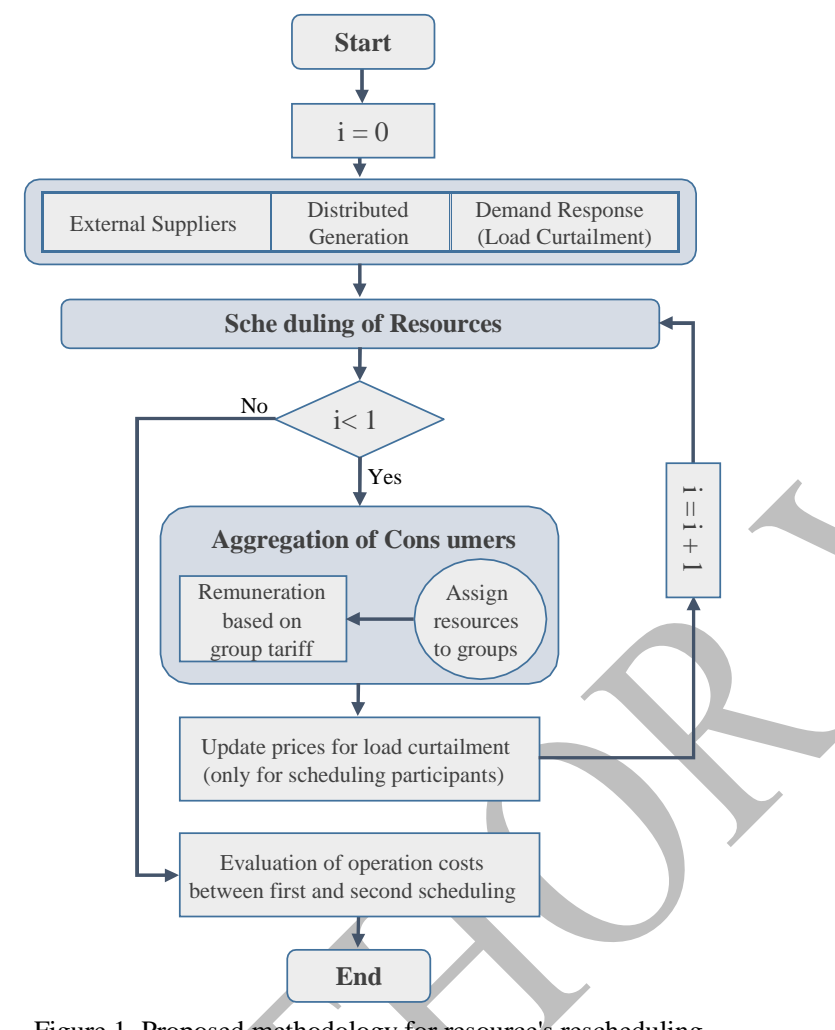

Figure 1. Proposed methodology for resource's rescheduling.

In this way, the aggregator defines new remuneration tariffs for the consumers participating in the curtailment demand response program, by aggregating these into groups, and defining for each one, an arithmetic average tariff based on the prices of the resources that belong to that group. Following this process, the aggregator updates the prices of the consumers for curtailment, and performs the scheduling of resources again, based on this data. It is important to notice that the resources who are not scheduled initially (contribution equal to 0 ), also are not considered for the aggregation so that these do not influence the group tariff. This allows for a more representative group tariff approach to the groups and for the resources belonging to it.

The scheduling of resources is based on an optimization problem, namely, a Mixed-Integer Linear Programming problem (MILP) developed in TOMLAB ${ }^{\mathrm{TM}} / \mathrm{TOMSYM}^{\mathrm{TM}}$ optimization environment. The mathematical formulation used for this is further detailed on section III - Mathematical Formulation. In what concerns the aggregation process, it is used the $k$-means clustering algorithm. This partition-based clustering algorithm guarantees a dynamic aggregation that hierarchical algorithms do not provide, since the chosen method allows for changes of objects between groups at each iteration of the clustering algorithm.

Clustering algorithms are based on the minimization of a given function at each iteration, i.e. the objective is to minimize the "distances" of objects to a given group center (defined or not by the user), such that the most closer objects to a certain group center are assigned to that group [15], [16]. In more detail, the center of the group changes between iterations, according to the objects that are assigned to it, providing an even more interesting approach to the clustering algorithm. In the present paper, the aggregation of resources is only possible for consumers participating in a curtailment demand response program, being these clustered based on their initially energy schedule and price. The remuneration process, that defines the group tariff, is based on a simple arithmetic average of the initial prices of the consumers for the load curtailment program.

In each period, it is identified and computed the number of resources, in this case consumers, that participate in the scheduling, and therefore are enabled for aggregation and remuneration processes. Neither the distributed generators and external suppliers are considered by the aggregator for aggregation, and consequently, remuneration tariffs. In this way, the aggregator can expect changes in the consumer's curtailment prices after aggregation and remuneration. This causes changes to the operation costs in each period, that the aggregator must evaluate, providing decision support on whether to perform aggregation or not of the resources, based on the operation costs obtained for each period.

Moreover, the proposed methodology can be used to facilitate the participation of the aggregator (and consequently, distributed energy resources) in the energy markets, since the clustering provides enough results for this. The clustering defines a given number of groups to the aggregator, containing a certain number of resources and building a summed amount of energy. Also, through the definition of a group tariff that remunerates all resources belonging to the group at the same price, provides the aggregator with the minimum bid tariff that it must obtain in market to avoid loss.

The present paper proposes a methodology to support the decision of an aggregator of resources in their management, minimizing operation costs through aggregation and remuneration methods that influence the optimization scheduling. In this way, by using clustering algorithms, it is possible to represent the aggregation of resources based on their initial energy schedule and price, and obtain summed amounts of energy corresponding to the several resources within a certain group.

\section{A. Scheduling Mathematical Formulation}

This section presents the mathematical formulation that models the optimization problem, as referred before. The optimization considers the resources available to the 
aggregator, namely, external suppliers (as generation sources outside of the region managed by the aggregator), distributed generators and consumers participating in the load curtailment demand response program. The objective function, as described in (1), minimizes the operation costs of the aggregator to maintain network balance, using the available resources. In this way, it is not considered revenues that the aggregator may obtain from supplying consumers or monthly payments that it receives for the energy management services that it produces.

$$
\begin{aligned}
& \text { Minimize } O C=
\end{aligned}
$$

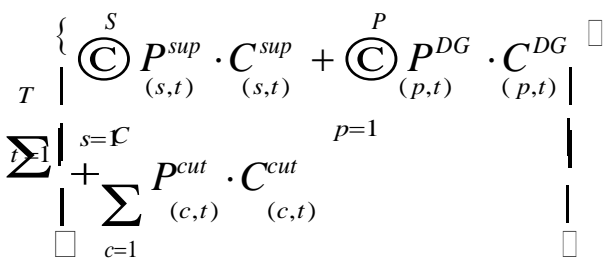

The balance of the network considers the contributions of each type of resource to face the consumption requirement, including the reduction made by the consumer's themselves in response to the load curtailment program. This is shown by equation (2), as follows.

$$
\begin{aligned}
& \sum_{s=1}^{S} P_{(s, t)}^{\text {sup }}+\sum_{p=1}^{P} P_{(p, t)}^{D G}=\sum_{c=1}^{C}\left(P_{(c, t)}^{\text {load }}-P_{(c, t)}^{c u t}\right) \\
& \forall t \in\{1, \ldots, T\}
\end{aligned}
$$

Technical limits of the resources must be considered by the aggregator so that no overloading can occur, causing an insufficient level of generation to meet demand. In this way, both for the external suppliers and distributed generators these are considered, by equations (3) and (4).

$$
\begin{aligned}
& P_{(s, t)}^{\min \sup } \leq P_{(s, t)}^{\text {sup }} \leq P_{(s, t)}^{\max \sup } \\
& \forall s \in\{1, \ldots, S\}, \forall t \in\{1, \ldots, T\} \\
& P_{(p, t)}^{\min D G} \leq P_{(p, t)}^{D G} \leq P_{(p, t)}^{\max D G} \\
& \forall p \in\{1, \ldots, P\}, \forall t \in\{1, \ldots, T\}
\end{aligned}
$$

Regarding the load curtailment demand response program, this is also limited by the availability of the consumer or a predefined step accorded between the consumer and the aggregator. This guarantees that the consumer's operation is not harmed when implementing the load curtailment program, since it is already established what can be curtail and its influence in the consumer's operation. The load curtailment program is detailed by equations (5) and (6).

$$
\begin{aligned}
& P_{(c, t)}^{\min c u t} \leq P_{(c, t)}^{c u t} \leq P_{(c, t)}^{\max c u t} \\
& P_{(c, t)}^{c u t}=P_{(c, t)}^{\max c u t} \cdot \lambda_{(c, t)}^{c u t}, \lambda_{(c, t)}^{c u t} \in\{0,1\}
\end{aligned}
$$

\section{B. Aggregation and Remuneration processes}

The aggregation process is performed using the $k$-means function of MATLAB ${ }^{\mathrm{TM}}$, which allows for a simple use of the corresponding clustering algorithm. The function takes as inputs a data vector or matrix and a $k$ number of clusters. The first parameter is related to the data that will be used to perform the clustering, thus it must insure that there is no evident pattern that may oversimplify the clustering and lead to obvious results. For instance, the type of resource or maximum standard capacity, are not adequate to serve as data input for the clustering algorithm, since these correspond to obvious classifiers that will lead to unjustified use of clustering algorithms. Moreover, the use of clustering algorithms intends to unveil the hidden patterns within the supplied data, and that will oblige the algorithm to iteratively compute the distances of the objects to the group centers' and assign them as these become closer to one. As mentioned in the previous section, the $k$-means clustering algorithm assigns, at each iteration, each consumer to a group, which changes the

center of the group. The center of the group can be a fixed value in some cases, or a dynamic one that depends upon the belonging objects. This latter approach is considered in the present paper, providing a dynamic movement of the objects amongst the several groups in each iteration, according to the group center. The second parameter is related to the number of clusters or groups that in this case the aggregator desires to form. The number of clusters must be equal or lower than the number of objects (i.e. number of participating consumers).

The present section details the case study implemented to verify the proposed methodology. The network considered is a

\begin{tabular}{|c|c|c|c|}
\hline $\begin{array}{c}\text { Generation } \\
\text { Resource } \\
\end{array}$ & $\begin{array}{c}\text { Inst. Power } \\
(k W)\end{array}$ & $\begin{array}{c}\text { Number of } \\
\text { units }\end{array}$ & $\begin{array}{c}\text { Price } \\
\text { (m.u./p.u.) } \\
\end{array}$ \\
\hline \multirow{5}{*}{ Photovoltaic } & 200 & 11 & \multirow{5}{*}{0,1560} \\
\hline & 150 & 9 & \\
\hline & 25 & 13 & \\
\hline & 20 & 24 & \\
\hline & 15 & 3 & \\
\hline Small hydro & 3010 & 1 & 0,1014 \\
\hline Biomass & 450 & 1 & 0,1231 \\
\hline Co-generation & 2100 & 1 & 0,0796 \\
\hline \multirow{4}{*}{ Wind } & 300 & 2 & \multirow{4}{*}{0,0964} \\
\hline & 200 & 2 & \\
\hline & 100 & 38 & \\
\hline & 20 & 11 & \\
\hline Total & 6590 & 116 & - \\
\hline External supplier & 10000 & 1 & Dynamic \\
\hline
\end{tabular}
180-bus distribution network, composed of 116 distributed generators, 90 consumers, and 1 external supplier. The distributed generators are divided upon five types: photovoltaic, small hydro, biomass, co-generation, and wind. These resource's features are demonstrated by Table I. Also, the external supplier is also detailed in the same table, where its price is considered dynamic.

TABLE I. GENERATION RESOURCES FEATURES

Regarding the consumers, these are also divided in five

types: domestic (DM), large commerce (LC), large industrial (LI), medium commerce (MC), and small commerce (SC). The curtailment capacity of each type of resource is shown in Figure 2, of which the large commerce offers more flexibility capacity followed by large industrial. In Figure 3, it is shown the individual curtailment tariff of each consumer, being that these remain the same through all periods. In fact, all tariffs 
remain constant throughout the periods except for the external supplier that has a dynamic pricing scheme.

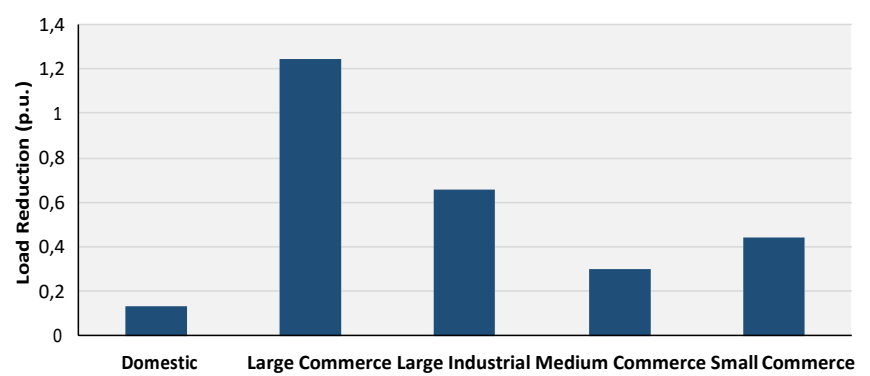

Figure 2. Total curtailment capacity for each type of consumer.

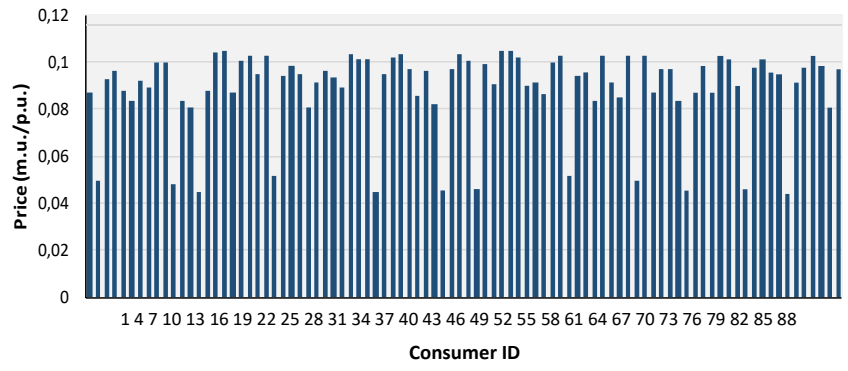

Figure 3. Energy price for each type of consumer.

The energy tariff that the external supplier considers for its dynamic pricing scheme, is based on four steps, namely, 0.1 (from period 24 to 5), 0.12 (in periods 6 to 8 , and from 18 to 23), 0.14 (from period 9 to 11 ), and 0.16 (from periods 12 to 17). According to this approach, the aggregator is more willing to use external suppliers in periods where the tariff is lower than other resources, for instance, except for the periods where the external supplier's tariff is at 0.16 , the photovoltaic units are more expensive than using the external supplier. The present section detailed the case study considered in this work, to verify the proposed methodology. The case study addresses the consideration of resources with distinct participation tariffs, providing some challenges to the optimization.

\section{RESULTS}

This section shows the results obtained for the case study regarding the proposed methodology. The first results obtained from the proposed methodology, are related to the initial scheduling, as shown in Figure 4. One can see that the major contributions come from distributed generators. Also, no consumer was left without supply, since the "Energy Nonsupplied" was not scheduled in any period.

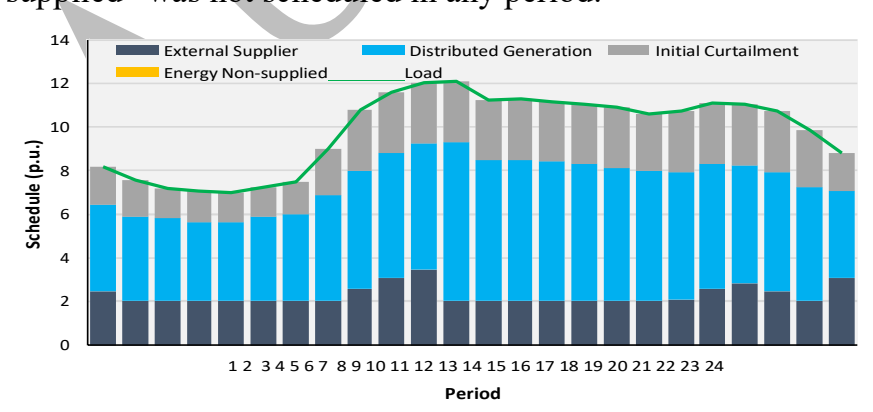

Figure 4. Initial scheduling of resources.
In Table II, it is presented the aggregation results obtained for the selected periods, 7 and 12. The first corresponds to the period where variations occurred in the energy curtailment schedule, while the latter corresponds to the period of peak consumption. The aggregation results are presented by type of consumer, demonstrating the distribution of consumer types amongst the groups.

\begin{tabular}{|c|c|c|c|c|c|c|c|}
\hline & $\begin{array}{c}\text { Resource } \\
\text { Type }\end{array}$ & 1 & 2 & 3 & 4 & 5 & Total \\
\hline \multirow{6}{*}{$\begin{array}{l}- \\
\overline{0} \\
0 \\
0\end{array}$} & Domestic & 7 & 0 & 1 & 4 & 1 & 13 \\
\hline & Large Industrial & 3 & 3 & 1 & 0 & 0 & 7 \\
\hline & Medium Commerce & 11 & 1 & 2 & 1 & 0 & 15 \\
\hline & Small Commerce & 21 & 1 & 3 & 1 & 0 & 26 \\
\hline & Large Commerce & 10 & 0 & 6 & 5 & 0 & 21 \\
\hline & Total & 52 & 5 & 13 & 11 & 1 & 82 \\
\hline \multirow{6}{*}{$\begin{array}{l}\frac{1}{2} \\
0 \\
0 \\
0 \\
0\end{array}$} & Domestic & 3 & 3 & 2 & 6 & 0 & 14 \\
\hline & Large Industrial & 2 & 1 & 1 & 1 & 2 & 7 \\
\hline & Medium Commerce & 3 & 1 & 0 & 10 & 2 & 16 \\
\hline & Small Commerce & 1 & 5 & 0 & 20 & 2 & 28 \\
\hline & Large Commerce & 6 & 3 & 2 & 9 & 2 & 22 \\
\hline & Total & 15 & 13 & 5 & 46 & 8 & 87 \\
\hline
\end{tabular}

Figure 5 presents the variation between the first and the second scheduling regarding the power scheduled (bars), and the changes in the price of scheduling (lines), for consumers participating in the demand response program of the aggregator. Figure 5 shows that in terms of energy, only in period 7 changes occur regarding the contribution of demand response for the aggregator's scheduling. In this case, the contribution of demand response increased in the second scheduling when compared with the first scheduling of period 7. This variation is caused by the modification of curtailment prices after the aggregation and remuneration processes. In terms of energy costs, Figure 5 shows that these suffer very slight variations in the first and second scheduling.

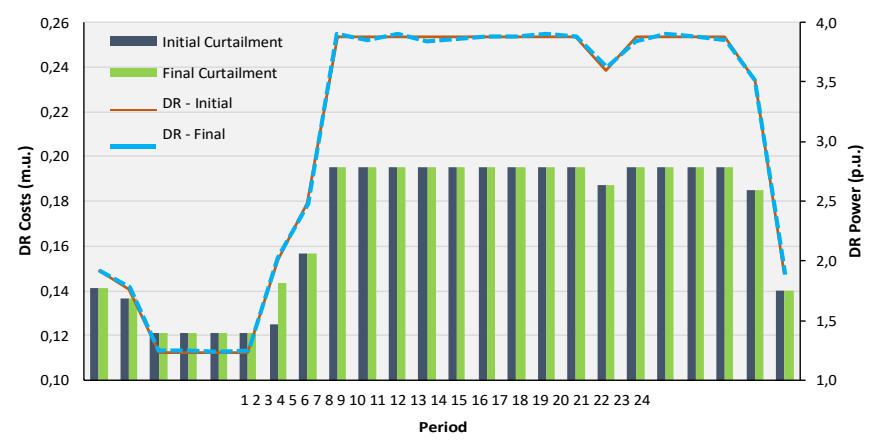

Figure 5. Differences between the initial and final scheduling of demand response, in terms of energy and cost.

In Figure 6 it is shown, for each individual consumer, the variation of scheduled energy curtailment, when comparing the second scheduling with the first. In this way, seven consumers raised their curtailment to contribute more to the aggregator's scheduling, in response to a variation in their load curtailment price. 


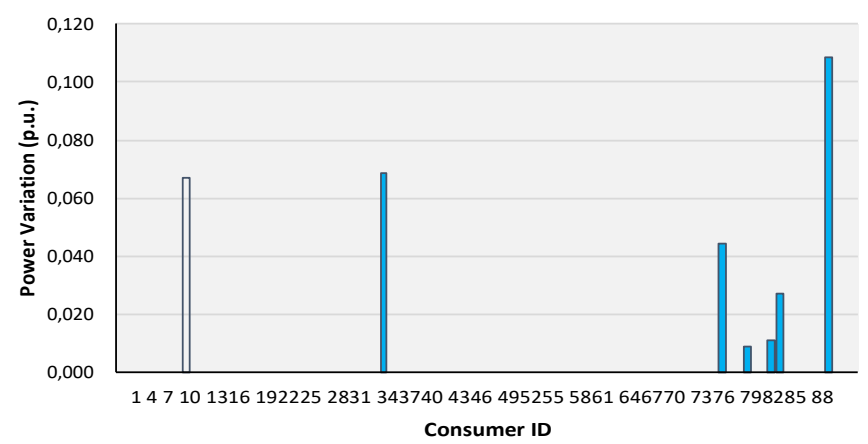

Figure 6. Load curtailment variation of the consumers, comparing the first with the second scheduling, in period 7 .

The variation of the load curtailment price for each consumer, is presented in Figure 7. Positive and negative variations reflect increases and decreases in comparison with the initial prices, respectively.

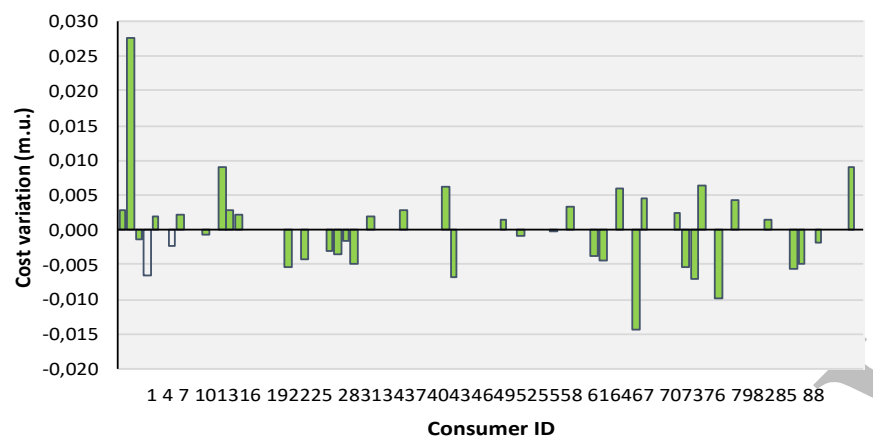

Figure 7. Load curtailment price variation of the consumers, comparing the first with the second scheduling, in period 7 .

Table III presents a detail of the results obtained for the consumers, in terms of variations observed in the power and price, in period 7. A total of six consumers are analyzed: the three with higher modification in their energy cost, and the three with higher modification in their energy curtailment schedule. Moreover, these consumers have the identification number $2,9,13,33,73$, and 87 .

TABLE III. DETAIL ON THE VARIATIONS OF CONSUMERS SCHEDULING

\begin{tabular}{|c|c|c|c|c|c|c|c|}
\hline & & \multicolumn{3}{|c|}{ Energy Cost } & \multicolumn{3}{|c|}{ Energy Curtailment } \\
\hline \multicolumn{2}{|c|}{ Type } & LI & $\mathrm{SC}$ & LI & $\mathrm{LC}$ & $\mathrm{LC}$ & $\mathrm{LC}$ \\
\hline \multicolumn{2}{|c|}{ ID } & 2 & 13 & 73 & 9 & 33 & 87 \\
\hline \multirow{2}{*}{$\stackrel{0}{0}$} & Initial & 0,1401 & 0,0179 & 0,1407 & 0,0000 & 0,0000 & 0,0000 \\
\hline & Final & 0,1401 & 0,0179 & 0,1407 & 0,0672 & 0,0687 & 0,1086 \\
\hline \multicolumn{2}{|c|}{ Diff. Power } & 0,0000 & 0,0000 & $\mathbf{0 , 0 0 0 0}$ & 0,0672 & 0,0687 & 0,1086 \\
\hline \multirow{2}{*}{$\stackrel{\mathscr{e}}{\underline{\underline{E}}}$} & Initial & 0,0494 & 0,0805 & 0,0869 & 0,0997 & 0,1002 & 0,1021 \\
\hline & Final & 0,0681 & 0,0893 & 0,0681 & 0,0997 & 0,1002 & 0,1021 \\
\hline \multicolumn{2}{|c|}{ Diff. Price } & $\mathbf{0 , 0 1 8 8}$ & 0,0088 & $-0,0188$ & 0,0000 & 0,0000 & 0,0000 \\
\hline
\end{tabular}

\section{CONCLUSIONS}

The present paper proposes a methodology for the rescheduling of resources by an aggregator. The resources are rescheduled based on the new tariffs obtained from aggregation and remuneration processes, namely, by implementing an arithmetic average group tariff for each of the groups obtained from the aggregation.

The results obtained, show that improvements can be made in terms of operation costs by implementing the proposed methodology. Moreover, the contributions of demand response can be raised considering the new prices, given the results obtained from the aggregation and remuneration processes. With this methodology, the aggregator can perform this analysis for each of the periods and decide in each one, on whether use the new prices obtained in the aggregation and remuneration processes, or maintain the initial ones. This is decided based on the variation of operation cost computed.

\section{REFERENCES}

[1] S. Rahnama, S. E. Shafiei, J. Stoustrup, H. Rasmussen, and J. Bendtsen, "Evaluation of Aggregators for Integration of Large-scale Consumers in Smart Grid," IFAC Proc. Vol., vol. 47, no. 3, pp. 18791885,2014

[2] H. Morais, T. Pinto, Z. Vale, and I. Praça, "Multilevel Negotiation in Smart Grids for VPP Management of Distributed Resources," IEEE Intelligent Systems, vol. 27, no. 6. pp. 8-16, 2012.

[3] J. Torriti, M. G. Hassan, and M. Leach, "Demand response experience in Europe: Policies, programmes and implementation," Energy, vol. 35, no. 4, pp. 1575-1583, 2010.

[4] E. Koliou, C. Eid, J. P. Chaves-Avila, and R. A. Hakvoort, "Demand response in liberalized electricity markets: Analysis of aggregated load participation in the German balancing mechanism," Energy, vol. 71, pp. $245-254,2014$.

[5] S. Ruggiero, V. Varho, and P. Rikkonen, "Transition to distributed energy generation in Finland: Prospects and barriers," Energy Policy, vol. 86, pp. 433-443, Nov. 2015.

[6] A. Rezaee Jordehi, "Allocation of distributed generation units in electric power systems: A review," Renew. Sustain. Energy Rev., vol. 56, pp. 893-905, 2016.

[7] S. Ø. Ottesen, A. Tomasgard, and S.-E. Fleten, "Prosumer bidding and scheduling in electricity markets," Energy, vol. 94, pp. 828-843, 2016.

[8] N. Mahmoudi, T. K. Saha, and M. Eghbal, "Modelling demand response aggregator behavior in wind power offering strategies," Appl. Energy, vol. 133, pp. 347-355, 2014.

[9] A. M. Carreiro, H. M. Jorge, and C. H. Antunes, "Energy management systems aggregators: A literature survey," Renew. Sustain. Energy Rev., vol. 73, pp. 1160-1172, 2017.

[10] D. P. Chassin and D. Rondeau, "Aggregate modeling of fast-acting demand response and control under real-time pricing," Appl. Energy, vol. 181, pp. 288-298, Nov. 2016.

[11] M. Paulus and F. Borggrefe, "The potential of demand-side management in energy-intensive industries for electricity markets in Germany," Appl. Energy, vol. 88, no. 2, pp. 432-441, 2011.

[12] P. Cappers, J. MacDonald, C. Goldman, and O. Ma, "An assessment of market and policy barriers for demand response providing ancillary services in U.S. electricity markets," Energy Policy, vol. 62, pp. 10311039, 2013.

[13] P. Faria, J. Spínola, and Z. Vale, "Aggregation and Remuneration of Electricity Consumers and Producers for the Definition of DemandResponse Programs," IEEE Transactions on Industrial Informatics, vol. 12, no. 3. pp. 952-961, 2016.

[14] J. Spínola, P. Faria, and Z. Vale, "Scheduling and aggregation of distributed generators and consumers participating in demand response programs," PowerTech, 2015 IEEE Eindhoven. pp. 1-6, 2015.

[15] R. Xu and D. Wunsch, "Survey of clustering algorithms," IEEE Trans. Neural Networks, vol. 16, no. 3, pp. 645-678, 2005.

[16] D. J. Vergados, I. Mamounakis, P. Makris, and E. Varvarigos, "Prosumer clustering into virtual microgrids for cost reduction in renewable energy trading markets," Sustain. Energy, Grids Networks, vol. 7, pp. 90-103, 2016. 